\title{
Compact Planar Sparse Array Antenna with Optimum Element Dimensions for SATCOM Ground Terminals
}

\author{
Junqi Lu ${ }^{1,2}$ and Yongxin Guo \\ ${ }^{1}$ School of Electronic Science and Engineering, National University of Defense Technology, Deya Road, Changsha 410073, China \\ ${ }^{2}$ Department of Electrical and Computer Engineering, National University of Singapore, Singapore 117576
}

Correspondence should be addressed to Junqi Lu; junqi.luke@gmail.com

Received 19 August 2015; Accepted 3 December 2015

Academic Editor: Jaume Anguera

Copyright (C) 2015 J. Lu and Y. Guo. This is an open access article distributed under the Creative Commons Attribution License, which permits unrestricted use, distribution, and reproduction in any medium, provided the original work is properly cited.

\begin{abstract}
A novel antenna array architecture for low-cost and compact SATCOM mobile terminal is presented. Based on equal-amplitude aperiodic phased array with fewer active chain numbers, it possesses advantages including lower weight, less cost, and higher power efficiency compared to conventional periodic phased arrays. It is implemented with printed patch antenna so that it guarantees compactness. The elements position and dimensions are jointly designed, with an effective sparse array synthesis strategy that takes actual patch antenna design constraint into consideration, to obtain a maximum array aperture efficiency. Executable and practical approach for variable dimension patch antenna designing, including defect substrate element and small scale array, is introduced and utilized to implement proposed sparse array. Full-wave simulation results demonstrate the advantages of proposed array antenna as well as the effectiveness of corresponding design approach.
\end{abstract}

\section{Introduction}

For the land and maritime satellite communication (SATCOM) terminals, especially the Communication-On-TheMove (COTM) application, the beam steering capability is a compulsory requirement to the antenna. Both mechanical and electrical beam steering technologies could be utilized to accomplish this task, but the latter has significant advantages including quicker response, lower profile, and light weight. Among all the electrical beam steering antennas, phased array antennas possess ultimate performance in terms of beam steering accuracy, beam shaping capability, and function flexibility. However, the extremely high cost of phased array restricted it from being widely applied in civilian and commercial areas. The cost of phased array is mostly brought in by the large number of active RF components (e.g., phase shifters, amplifiers) within it. Though some classic methods for reducing the number of active components in phased array have been developed, such as subarraying, these approaches are not applicable when wide angle beam steering is required.

Sparse array with fewer active control numbers provides an alternative way to reduce the cost of phased array. It has been demonstrated that an array with fewer radiating elements located aperiodically on the array aperture could exhibit comparable performance in terms of pattern shaping and side lobe suppression [1-3]. Another advantage of sparse array is that it is possible to use an equal-amplitude excitation instead of conventional tapering excitation. In this case, all the power amplifiers in the active chains could operate at their best efficiency point, thus maximizing the DC-RF efficiency [4]. The design methods of sparse array were firstly introduced in the 1960s $[5,6]$ and got rapidly developed in recent years with the aid of advance optimization algorithms $[7,8]$ and high efficiency EM computing techniques. A series of problems on the design of a sparse array have been thoroughly discussed in the past few years, including pattern synthesis $[9,10]$, mutual coupling effects consideration and tackling [11, 12], and novel sparse/thinned strategies [13-15]. However, as intrinsic drawbacks, the gain of sparse array would decrease roughly proportional to the reduction of number of radiating elements [16] and a strict sidelobe level (SLL) requirement is hard to be accomplished for sparse array especially in scanning situation [17]. These shortcomings might explain the reason why sparse array antenna has never been put into practical use in SATCOM terminals till now, 
except for few demonstration systems developed by ESA which might still be far from real application [18].

The poor aperture efficiency is a fundamental limitation of sparse array if identical elements are used, especially when low SLL is required [19]. To counteract these limitations, the size of each element could be introduced as an additional design parameter for sparse array besides the position and excitation of elements $[14,19,20]$. The design principle, strategy, and radiating performance of sparse array with jointly optimized element positions and dimensions have been thoroughly discussed in $[19,21]$. Compared to traditional equalamplitude excitation sparse array with isotropic or identical elements, it was shown in [19] that a sparse array with elements which have different aperture dimensions exhibits much better aperture efficiency, and $2.1 \mathrm{~dB}$ gain increasing could be obtained. An accurate and complete design approach was also introduced in this literature. However, there are still some problems left to be discussed and tackled. Firstly, the proposed method in $[19,21]$ is based on the utilization of ideal "max efficiency" elements which are not actually achievable and therefore degraded its effectiveness. Secondly, the indicated research background in [19] is the antenna for space platforms, especially the GEO SATCOM satellite for which only a limited scanning angle is required. In this case, the proposed method is demonstrated with a sparse array consisting of horn antennas whose aperture dimensions vary from $2 \lambda$ to $3.4 \lambda$. It could be inferred that a wide angle beam steering with low SLL is hardly to be accomplished with this array configuration. We also found in [19] that this configuration makes it easier to conduct synthesis procedure compared to those more typical phased arrays whose minimum element aperture is usually not larger than $0.7 \lambda$. On the other hand, the using of horn antennas prevented the implementation of a low profile antenna array. Although it was mentioned in the literature that other kinds of antennas such as printed patches could be used as array element solutions, no further discussions were provided on how to modify the array synthesis method according to the actual radiating properties of those antennas, rather than the ideal "max efficiency" model.

In this paper a high efficiency sparse array synthesis method is introduced and utilized to design a sparse array with patch antenna. A simple but effective defect substrate technology is utilized to implement patch antennas with different aperture dimensions while working in the same frequency. With proposed synthesis method and diverse patch antenna elements, an annular sparse array is designed and has its performance evaluated and compared with those of traditional periodic array and sparse array.

This paper is organized as follows. In Section 2 the high aperture efficiency annular sparse array synthesis principle is briefly introduced. In Section 3 the designing of a high efficiency annular sparse array with dimension variable square aperture antenna elements is conducted. The design of an annular sparse array based on different kinds of patch antenna elements is carried out in Section 4. In Section 5 the performance of proposed sparse array is analyzed and some conclusion derived.

\section{High Aperture Efficiency Sparse Array Synthesis Principles}

It has been proven that the relationship between the excitation and the radiation pattern of two particular antenna arrays could be formulated as follows [19]:

$$
\begin{aligned}
& \int_{0}^{\infty} \frac{1}{\left(k_{0} u\right)^{2}}\left|F_{0}(u)-F_{u}(u)\right|^{2} u d u \\
& =\left(\frac{2 \pi}{k_{0}}\right) \int_{0}^{\infty} \frac{1}{\rho^{2}}\left|I_{0}(\rho)-I_{u}(\rho)\right|^{2} \rho d \rho \\
& k_{0}=\frac{2 \pi}{\lambda_{0}}, u=\sin (\theta),
\end{aligned}
$$

where $F_{0}(u)$ and $I_{0}(u)$ are the reference pattern and excitation cumulative function; $F_{u}(u)$ and $I_{u}(u)$ are the pattern which intends to imitate the reference and its excitation cumulative function.

It is therefore logical to conduct the sparse array synthesis with the strategy of imitating the excitation cumulative function of the reference target. The common density tapering method for sparse array synthesis is a specific case of this strategy. On the other hand, instead of calculating the complete radiation pattern of particular excitation for assessing the array synthesis results, one can take the cumulative function of the excitation as the evaluating object. Compared to the calculation of radiation pattern which might be time-consuming to some extent, the handling of cumulative function is much more effective.

The synthesis principle could be graphically interpreted and shown in Figure 1. By utilizing a joint position and dimension adjustment on the excitation, the cumulative function could be closer to the reference function. Therefore according to (1), the corresponding radiation pattern could be more similar to what is expected. Detailed introduction on this issue has been given in $[19,21]$.

\section{Synthesis Method of Annular Sparse Array with Patch Antenna}

For the COTM terminal antennas, there is no specific preferred direction in the whole upper hemisphere space. Therefore a circle could be viewed as the most suitable outline of the proposed phased array. Meanwhile, for the sake of pattern symmetries and implementation simplicity, annular configuration of the elements is preferable to rectangular or triangular grids configurations. Therefore the design will be focused on annular arrays.

For the purpose of reducing the profile and cost of proposed sparse array, compact radiating element should be utilized instead of bulky ones such as horn or waveguide. Printed patch is a maturely developed antenna with advantages including low profile and low fabrication cost. It has been widely used in COTM terminal antennas in recent years $[18,22]$, thus demonstrating its feasibility in SATCOM application. For these reasons, printed patch is chosen as the radiating element for the proposed sparse phased array. 


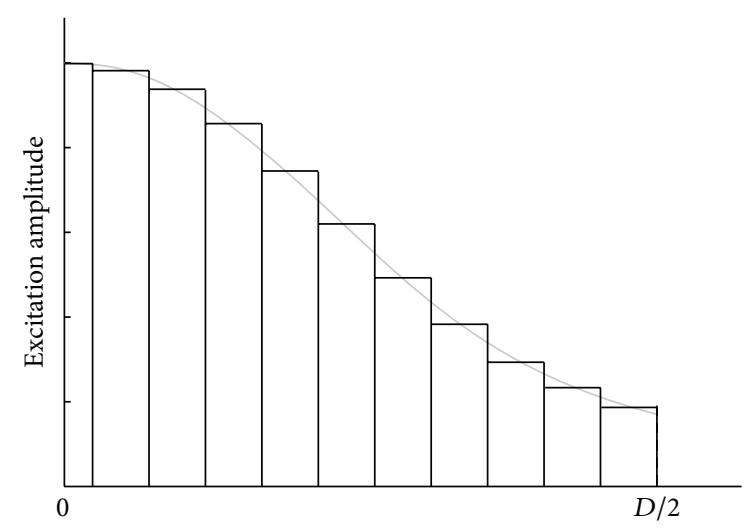

Array aperture
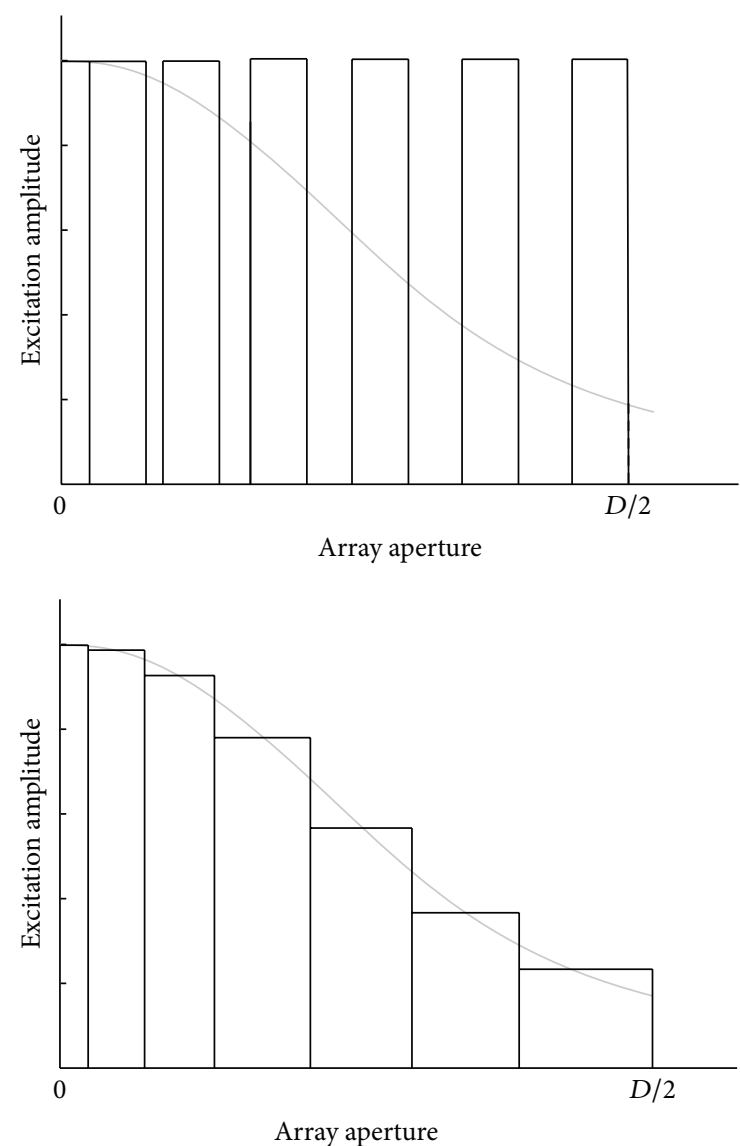
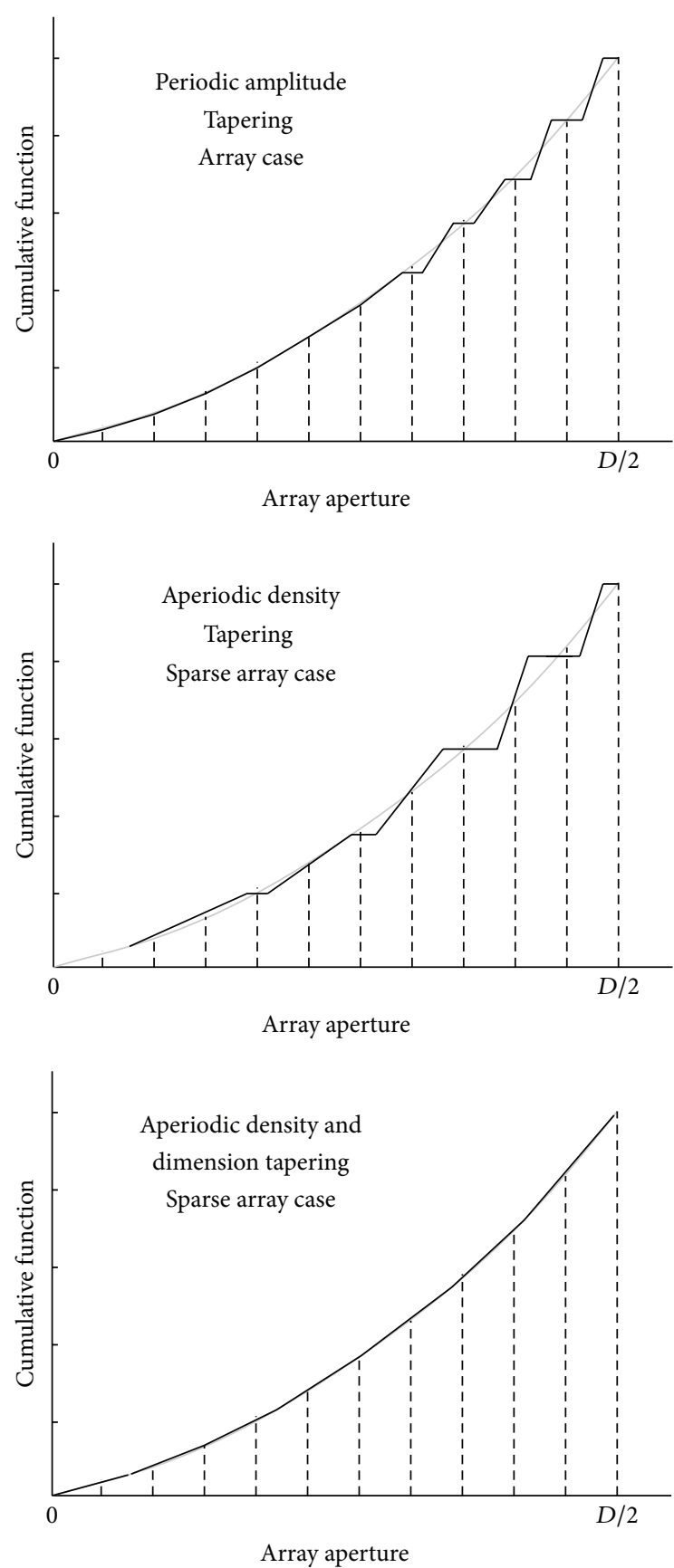

FIGURE 1: High aperture efficiency sparse array synthesis principle.

In order to apply the proposed element position and dimension joint synthesis approach, a prerequisite is to determine the aperture dimension of patch antenna. In this paper, the dimension is defined as the edge length of a virtual square radiating aperture. This virtual square could also be viewed as the effective aperture of patch antenna and could be obtained from the gain with the following equation, in which $D_{\text {patch }}$ denotes the aperture dimension, $A_{\text {eff }}$ denotes the virtual square or effective aperture, and $G_{\text {patch }}$ and $\lambda$ represent the gain and operating wavelength of the patch antenna:

$$
D_{\text {patch }}=\sqrt{A_{\text {eff }}}=\sqrt{\frac{G_{\mathrm{patch}} \cdot \lambda^{2}}{4 \pi}} .
$$

The patch antenna could be roughly viewed as a square aperture and the aperture dimension is to be determined by (2). To synthesize a high efficiency annular sparse array with 


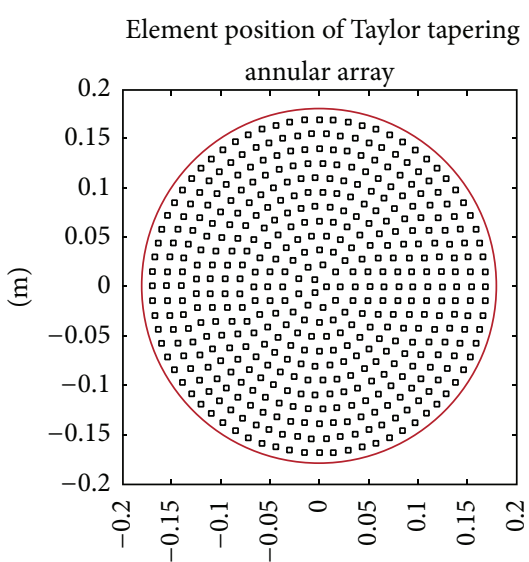

$(\mathrm{m})$

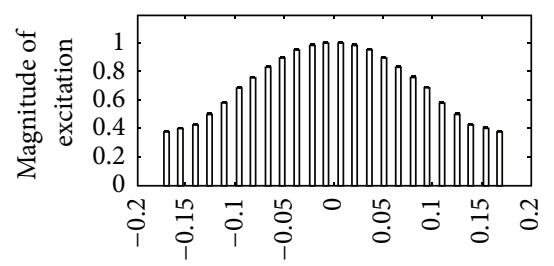

Ring diameter (m)

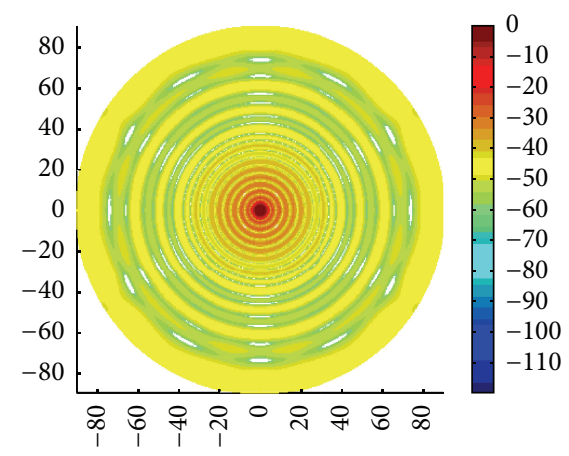

Element position of conventional sparse

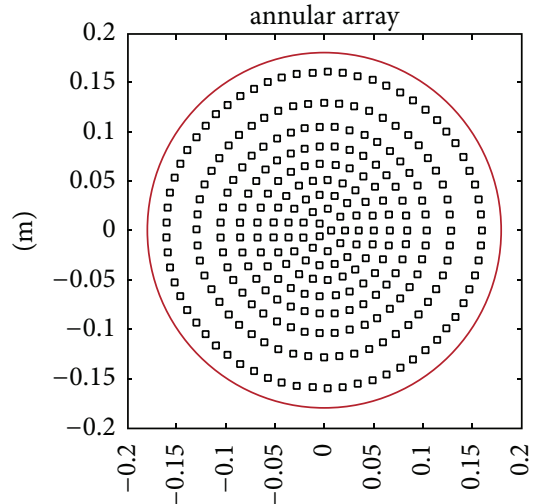

$(\mathrm{m})$

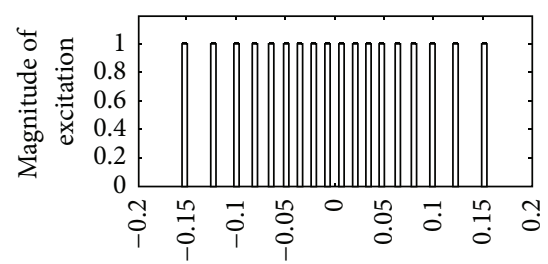

Ring diameter $(\mathrm{m})$

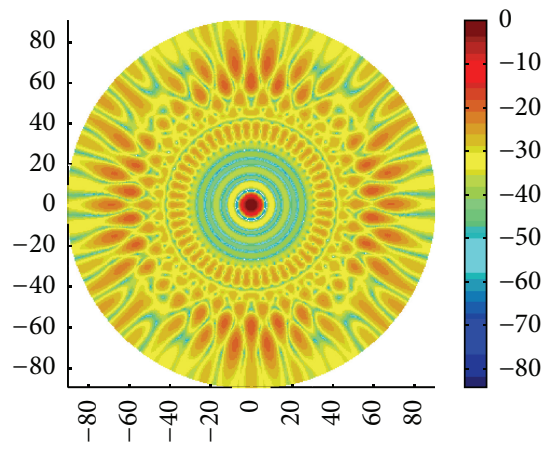

Element position of novel sparse

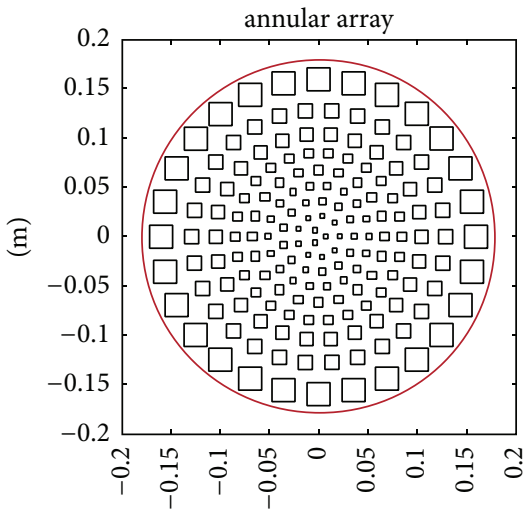

(m)

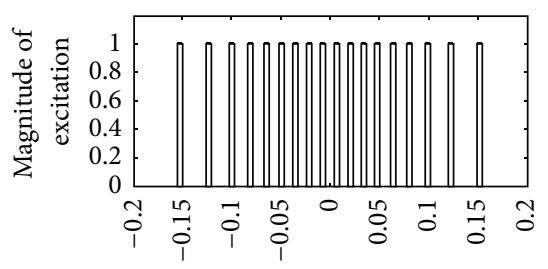

Ring diameter $(\mathrm{m})$

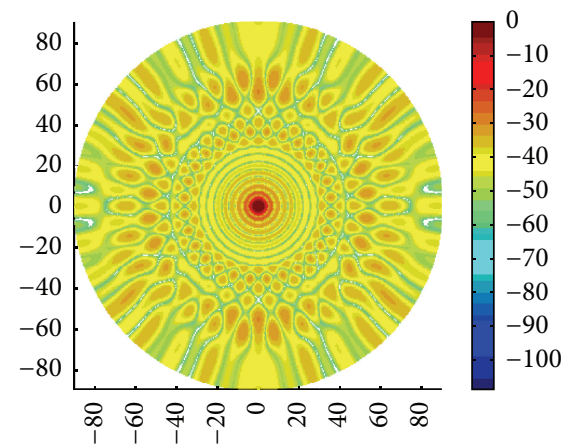

FIGURE 2: Synthesis results of annular periodic array (left column), conventional sparse array (middle column), and proposed high efficiency sparse array (right column). The array elements distribution (upper row), excitation distribution (middle row), and radiation pattern (lower row) are illustrated.

square aperture elements, the first step is to obtain the width of each coaxial ring area of the proposed annular sparse array, according to the method thoroughly introduced in [19]. Once the width of each ring is obtained, then the dimension of the square aperture elements to be populated in the ring areas could be determined, as a portion close to but not reaching 1, for example, 0.8 or 0.9 , of the ring width. Each ring area should be populated by as many elements as possible without overlap. All square apertures should be located on the circle which represents the geometric center of the ring area.

A set of illustrative synthesis results are shown in Figures 2 and 3 and Table 1 . A $-25 \mathrm{~dB}$ SLL requirement is achieved by a periodic annular array, a conventional density tapering array, and the proposed high efficiency sparse array. It could be noticed in Figure 2 that although having only about $30 \%$ of the number of elements, the proposed sparse array exhibits an almost identical aperture efficiency compared to
TABLE 1: Syntheiss results of three kinds of annual arrays.

\begin{tabular}{lccc}
\hline & $\begin{array}{c}\text { Periodic } \\
\text { array }\end{array}$ & $\begin{array}{c}\text { Conventional } \\
\text { sparse array }\end{array}$ & $\begin{array}{c}\text { High } \\
\text { efficiency } \\
\text { sparse array }\end{array}$ \\
\hline Number of elements & 452 & 270 & 169 \\
Directivity (dBi) & 33.98 & 31.90 & 33.62 \\
SLL (dB) & -25.9 & -24.95 & -25.2 \\
Aperture efficiency & $86.64 \%$ & $53.64 \%$ & $79.56 \%$ \\
\hline
\end{tabular}

periodic array. This performance is much better than that of a conventional annular sparse array with only one kind of element. The SLL requirement is well fulfilled with all the three arrays, yet the performance of proposed array is obviously better than that of traditional sparse array, as shown in Figure 3. 


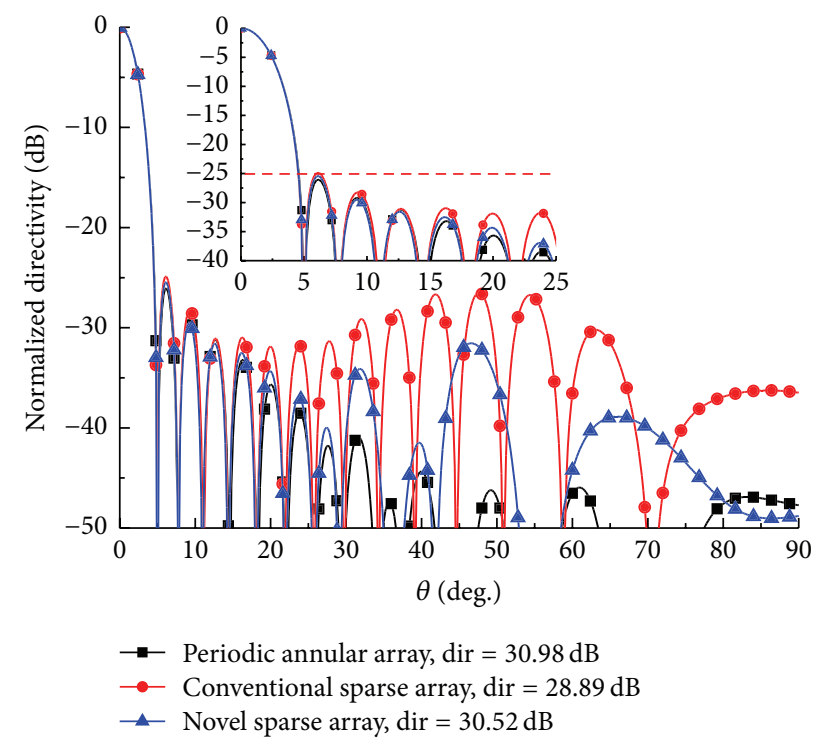

FIGURE 3: Pattern synthesis results of $-25 \mathrm{~dB}$ SLL pattern.

\section{Design Results of a Compact Annular Sparse Array with Patch Antenna}

Based on the array synthesis results introduced above, a high efficiency compact annular sparse array is designed. The design work consists of (1) designing of printed patch antennas having different aperture dimensions and (2) designing of annular sparse array with those patch antennas. The demonstrating frequency is chosen at $14.25 \mathrm{GHz}$, which is a typical value of practical SATCOM uplink band.

\subsection{Design of Patch Antennas Having Different Apertures.} According to the array synthesis results, the required aperture dimensions vary from $0.6 \lambda$ to $1.61 \lambda$. Two strategies are utilized to achieve this requirement, that is, simple defect substrate patch and $2 \times 2$ arrays.

4.1.1. Defect Substrate Patches for Aperture Dimensions Not Larger Than $0.9 \lambda$. Unlike real aperture antennas such as the horn antenna introduced in $[19,23]$ whose physical aperture could be easily defined and continually adjustable, printed patch antennas exhibit a much more strict constraint on the dimension of the antenna aperture. In general, the size of patch antenna $D_{\text {patch }}$ should be about half of the effective wavelength $\lambda_{\text {eff }}$, which is mostly determined by the effective dielectric constant $\varepsilon_{\text {eff }}$, thickness of substrate $t_{\text {sub }}$, and a modification factor $\kappa_{\text {structure }}$ which represents the structure effects (e.g., multilayer or inhomogeneous substrate and superstrate):

$$
D_{\text {patch }} \approx \frac{\lambda_{\text {eff }}}{2}=\frac{\lambda_{0} / \varepsilon_{\text {eff }}}{2}, \quad \varepsilon_{\text {eff }} \sim\left\{\varepsilon_{\text {sub }}, t_{\text {sub }}, \kappa_{\text {structure }}\right\} .
$$

In order to adjust the effective aperture of printed patch antenna without affecting the operating band, one executable solution is to modify the effective dielectric constant of the substrate. Several approaches could be used to achieve this

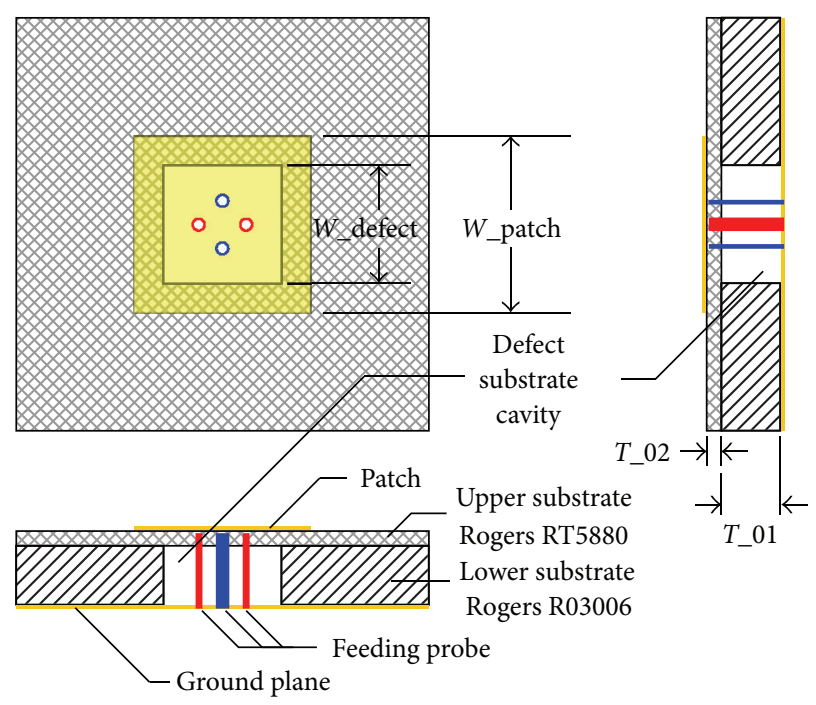

Figure 4: Sectional view of defect substrate patch antenna.

purpose while the defect substrate technology would be an optimal choice. By removing part of the substrate between patch and ground plane, the effective dielectric constant could be varied within a particular range: specifically, (1) high effective dielectric constant, if no substrate is removed; (2) moderate effective dielectric constant, if part of substrate is removed (defect); and (3) low effective dielectric constant, if almost all substrate is removed. The technology is shown in principle in Figure 4.

The proposed defect substrate patch antenna consists of five parts: (1) the printed radiating patch, (2) the upper substrate to supporting patches, (3) the lower substrate patch in which defect cavity is constructed to adjust the aperture dimension of antenna, (4) differential feeding probe to provide a low cross-polarization radiation, and (5) ground plane. The upper substrate should be much thinner than the lower substrate to maximize the adjusting efficiency of effective dielectric constant via defect substrate technology. The two-layer substrate structure makes it very easy to proceed substrate removal work. For a joint consideration of electrical and mechanic performance as well as fabrication feasibility, the material of lower substrate is chosen as Rogers R0306 $\left(\varepsilon_{r}=6.15\right)$ and the thickness T_01 is $0.5 \mathrm{~mm}$; the material of upper substrate is chosen as Rogers RT5880 $\left(\varepsilon_{r}=\right.$ 2.2) and thickness T_02 is $0.127 \mathrm{~mm}$.

The primary parameter in the design of defect substrate patch antenna is the defect factor $K_{-}$defect, which could be easily defined as the ratio between the width of subdefected cavity $W_{-}$defect and radiating patch $W_{-}$patch. The value of $K \_$defect ranges from 0 to a particular positive number. Theoretically the aperture of patches could be modified continually by changing $K \_$defect. However, the patch dimension would become very sensitive to the changing of the size of defect substrate cavity when the value of $K_{-}$defect is getting close to 1 . This nonlinear response will bring in unnecessary difficulties in fabrication. In this case, only three types of defect substrate patch antenna are designed: (1) complete 


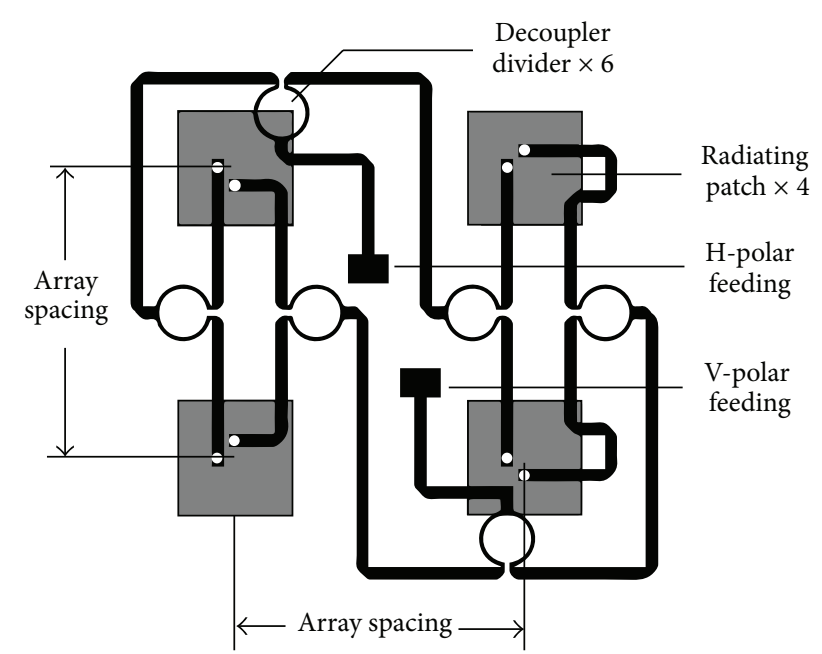

Figure 5: Pairwise mirrored element array.

TABLe 2: Design parameters of simple defect substrate patch antenna.

\begin{tabular}{lccc}
\hline & Element 1\# & Element 2\# & Element 3\# \\
\hline$W$ _patch $(\mathrm{mm})$ & 3.95 & 6.9 & 9.0 \\
$W$ _defect $(\mathrm{mm})$ & 0 & 7.0 & 12.0 \\
Aperture size $(\mathrm{mm})$ & 12.8 & 15.2 & 18.3 \\
Directivity & 4.62 & 6.52 & 9.46 \\
\hline
\end{tabular}

TABLE 3: Design parameters of proposed element array.

\begin{tabular}{lccc}
\hline & Element 4\# & Element 5\# & Element 6\# \\
\hline Components & Element 1\# & Element 2\# & Element 3\# \\
Array spacing $(\mathrm{mm})$ & 10.0 & 12.0 & 14.0 \\
Aperture size $(\mathrm{mm})$ & 25.9 & 28.8 & 33.8 \\
Directivity & 12.57 & 21.04 & 32.48 \\
\hline
\end{tabular}

substrate ones with $12.8 \mathrm{~mm}$ aperture size (element $1 \#)$; (2) partially defective substrate ones with $15.2 \mathrm{~mm}$ aperture size (element 2\#), and (3) fully defective substrate ones with $18.3 \mathrm{~mm}$ aperture size (element $3 \#)$ (Table 2).

4.1.2. Small Arrays for Aperture Dimensions Larger Than $0.9 \lambda$. The maximum available aperture dimension of a simple defect substrate patch is about $0.9 \lambda$. For larger apertures requirement, small scale arrays could be adopted. For the consideration of symmetries of radiating patterns and the maximum aperture size constraint, only a $2 \times 2$ array configuration is suitable for this purpose.

In order to guarantee a low cross-polarization level, a pairwise mirrored elements configuration which was introduced in [24] is utilized. The array fabrication and feeding network is shown in Figure 5.

For the sake of fabrication simplicity, three types of arrays are designed, with the aperture dimensions (1) $25.9 \mathrm{~mm}$; (2) $28.8 \mathrm{~mm}$; and (3) $33.8 \mathrm{~mm}$ (Table 3). Three different elements

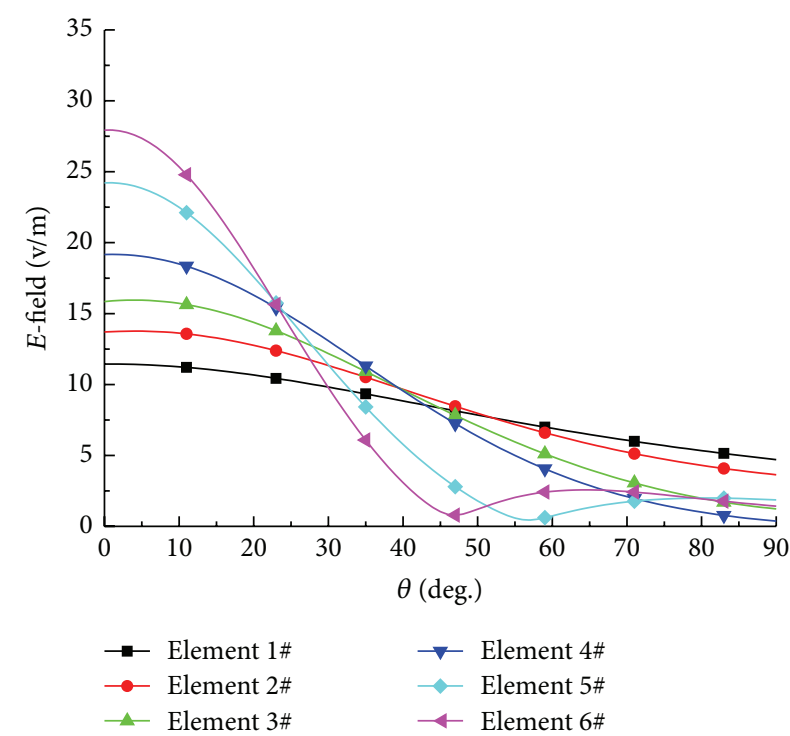

Figure 6: E-field pattern of designed elements.

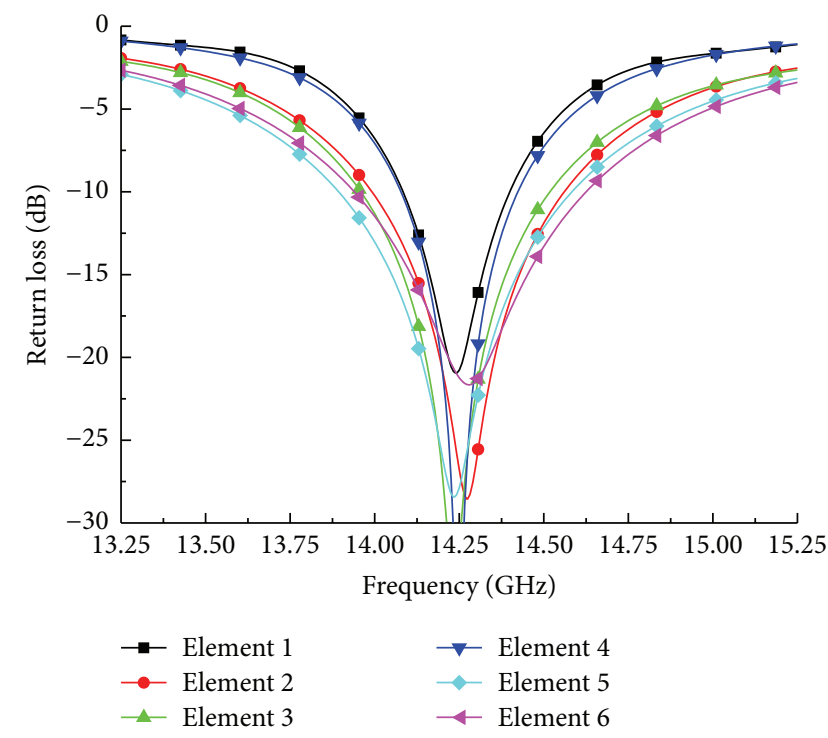

Figure 7: Return loss of designed elements.

designed in the last section are used in the design of these three arrays.

The performance and design results of elements 1-6\# are illustrated in Figures 6 and 7. It could be verified that the $E$-field amplitude in the boresight direction of each element is roughly proportional to its aperture dimension, and all elements' operating frequency locates around $14.25 \mathrm{GHz}$ although with different aperture dimensions.

4.2. Design of High Efficiency Sparse Array with Patch Antenna Elements. Based on the array synthesis and element design results obtained, an entire high efficiency sparse array is modeled and simulated using CST MW Studio. The 1st and 2nd rings of elements near the center are implemented with 


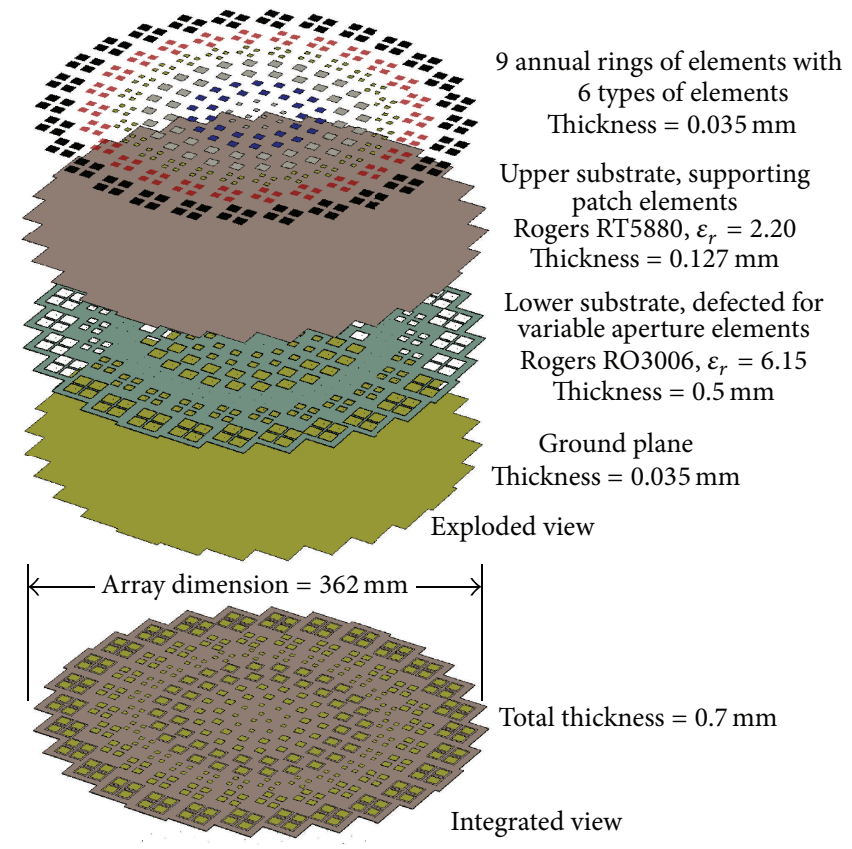

FIGURE 8: Detailed structure of proposed sparse array.

TABLE 4: Simulation results of three types of arrays.

\begin{tabular}{lccc}
\hline & $\begin{array}{c}\text { Periodic } \\
\text { array }\end{array}$ & $\begin{array}{c}\text { Conventional } \\
\text { sparse array }\end{array}$ & $\begin{array}{c}\text { High efficiency } \\
\text { sparse array }\end{array}$ \\
\hline Number of elements & 452 & 270 & 169 \\
Directivity (dBi) & 33.3 & 31.1 & 33.2 \\
SLL (dB) & -26.5 & -21.6 & -24.2 \\
Aperture diameter & $362 \mathrm{~mm}$ & $362 \mathrm{~mm}$ & $362 \mathrm{~mm}$ \\
Aperture efficiency & $73.26 \%$ & $44.15 \%$ & $71.60 \%$ \\
\hline
\end{tabular}

element 1\#; the 3rd and 4th rings are implemented with element 2\#; the 5th and 6th rings are implemented with element $3 \#$. The 7 th, 8 th, and 9 th rings are implemented with array elements $4 \#, 5 \#$, and $6 \#$, respectively. The total number of independently excited elements is 143 .

The array overall structure is the combination of all the elements and could be divided into 4 parts just like each element: (1) the radiating patches, (2) the upper substrate which is mainly for supporting patches above, (3) the defected lower substrate to implement aperture adjustment, and (4) the ground plane. The total thickness is only $0.7 \mathrm{~mm}$ or $0.033 \lambda_{0}$ (the feeding net layer excluded). Figure 8 shows the detail of the array model. It is clear that a common PCB technology could be easily utilized to fabricate this array without any complicated procedure.

The characteristics and performance are summarized in Table 4. The most noticeable point is that the SLL of sparse arrays is not achieved. Our research shows that it is due to the complicated mutual coupling effects.

\section{Technical Results and Discussion}

Since all the elements' excitation amplitudes are identical in the proposed sparse array, all power amplifiers could be operating in their best efficiency point, thus maximizing the overall DC-RF efficiency. Taking a typical power amplifier DC-RF efficiency curve as shown in Figure 10 as an example [4], it could be noticed that the lower the amplifier output, the lower the DC-RF efficiency.

The equivalent isotropically radiated power (EIRP), which is the product of antenna gain and input RF power, is calculated and assessed among three types of annular arrays and the results are summarized in Figure 11. The values of periodic array are taken as reference and values of others are proportionally drawn in the figure. The most noticeable points in the figure are that, (1) with much fewer elements, the proposed sparse array exhibits an almost identical directivity as the periodic array and, (2) by using density tapering instead of amplitude tapering, the DC-RF efficiency is doubled. As for the EIRP value it is logical to obtain a significant increase.

Beam scanning capability is a key performance requirement of COTM terminal antenna. The scanning performance of proposed sparse array is simulated and assessed; some results are illustrated in Figure 12.

The maximum grating-lobe free scanning angle is about 55 degrees with respect to the boresight direction. A $-6 \mathrm{~dB}$ gain beam could be achieved at about 35-degree scanning angle and $-10 \mathrm{~dB}$ gain beam obtained at about 45 degrees. Compared to the periodic annular array, of which there is no grating-lobe in the entire upper hemisphere, the -6 and $-10 \mathrm{~dB}$ scanning angles are 55 and 73 degrees, respectively. For the conventional annular sparse array consisting of 

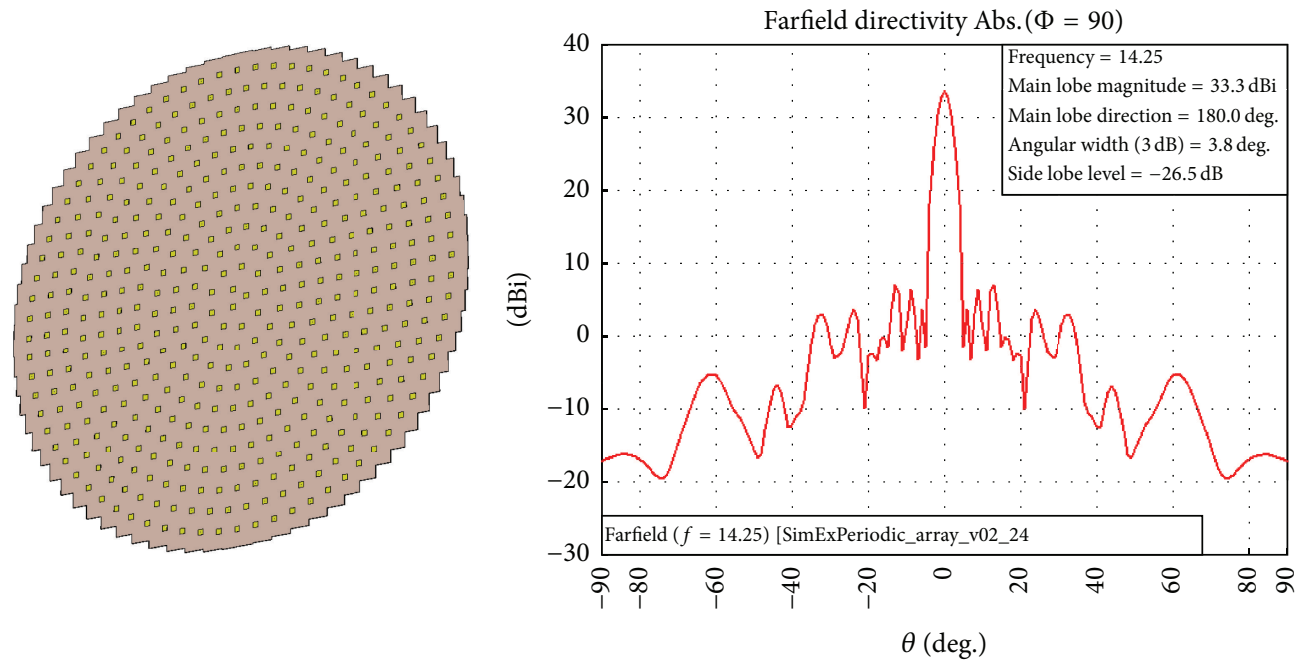

(a)
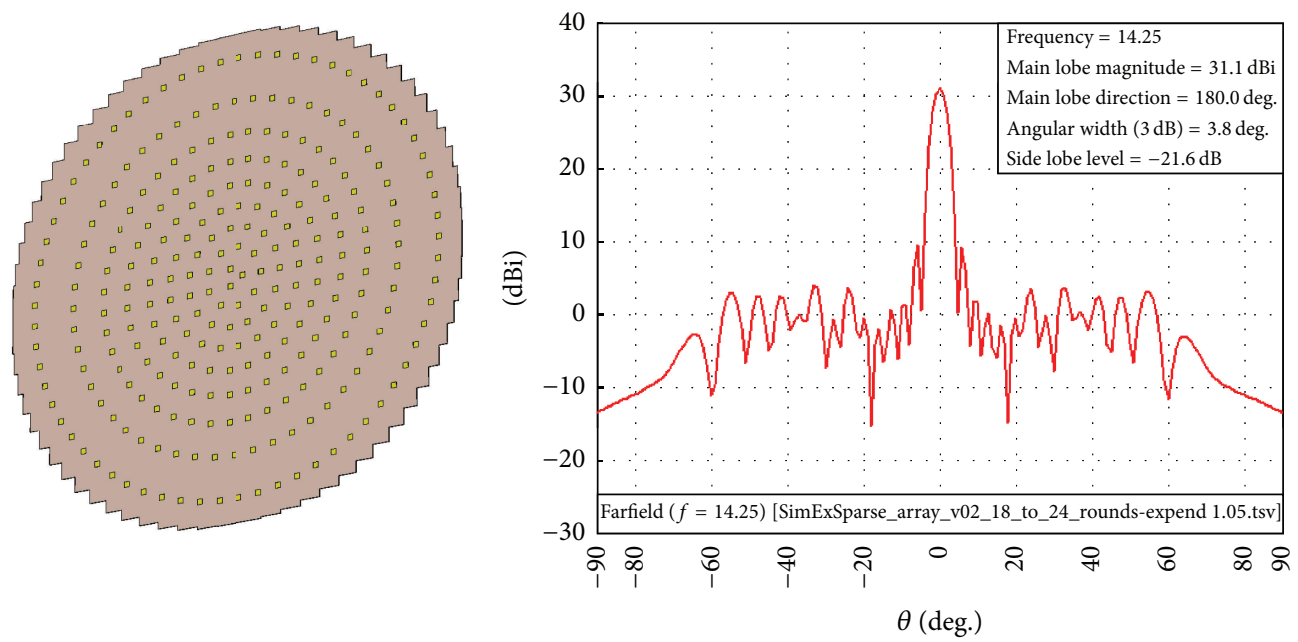

(b)
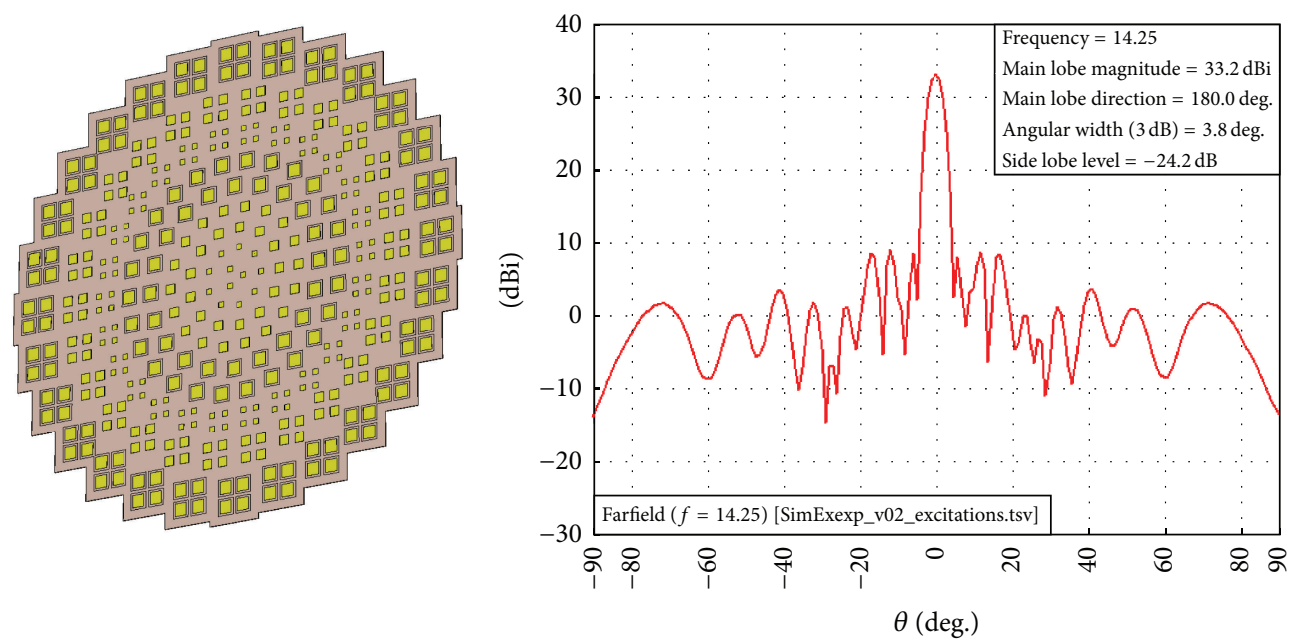

(c)

FIGURE 9: Simulation model and results of three arrays: (1) periodic annular array (upper), (2) conventional sparse array (middle), and (3) high efficiency sparse array (lower). 


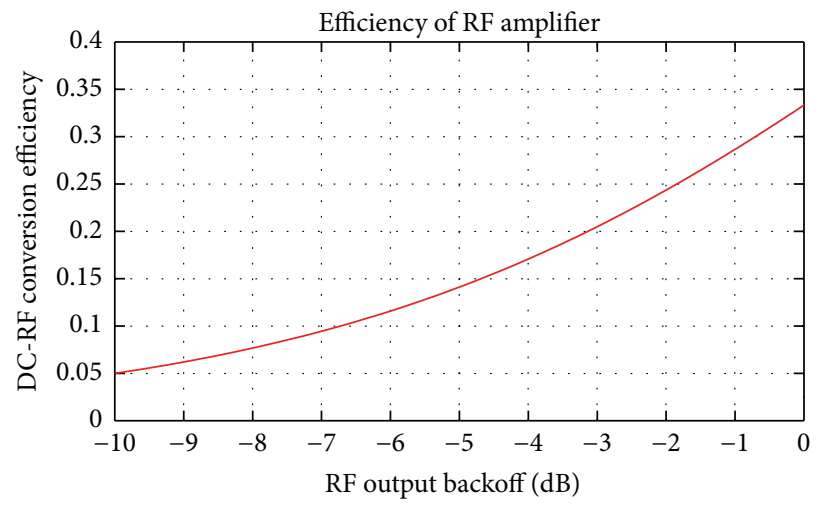

Figure 10: Power amplifier DC-RF conversion efficiency.

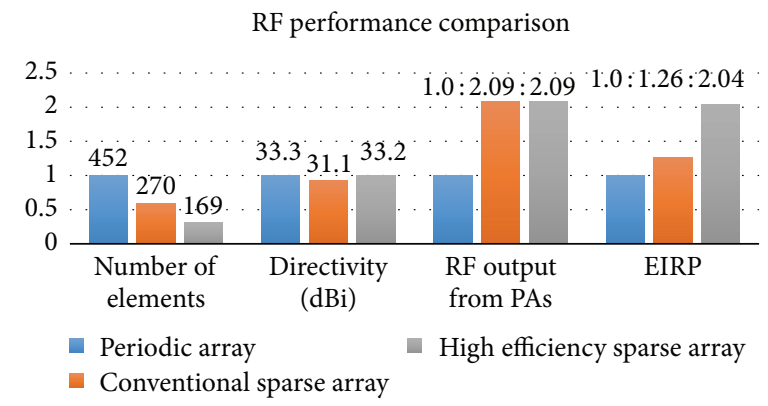

FIGURE 11: RF performance comparison.

identical radiating elements, there is also no grating-lobe in the whole visible area, and the $-6 \mathrm{~dB}$ beam scanning angles are 58 degrees; $-10 \mathrm{~dB}$ beam scanning angle almost reaches the horizontal direction.

Apparently, for the proposed sparse array, the beam scanning performance is not optimal, and that might limit its global application in COTM terminals. This problem could be inferred from the intrinsic drawback of density tapering array [5]. Nevertheless, considering the advantage of the proposed sparse array in DC-RF efficiency and EIRP level, it is still a promising solution for COTM ground and maritime terminals, if the deploying area is restricted in the low latitude and tropical regions where the zenith angle of GEO communication satellite will not be greater than 30 or 40 degrees, especially for those steady moving platforms such as trains or ships, or vehicles running in urban area or on expressways.

As shown in Figure 13, the gain of beam steering to 30 degrees with respect to zenith direction is $26 \mathrm{dBi}$, comparable to that of conventional sparse array. Though $2.9 \mathrm{~dB}$ lower than the gain of the beam of periodic array in the same scanning situation, the final EIRP value would be predicted to be almost the same since the gap could be filled up by a $3 \mathrm{~dB}$ DC-RF efficiency gain. It could also be noticed that the side lobe near main beam is always kept in a low level as the beam scanning - a favorable characteristic of SATCOM.

\section{Conclusion}

An effective design strategy of high efficiency sparse array antenna has been introduced. Both elements' position and dimension are utilized as synthesis parameters and optimized simultaneously. Both analytical and full-wave simulation results demonstrate the effectiveness and accuracy of the introduced method (Figures 2 and 9 and Tables 1 and 4). The proposed printed patch antenna based sparse array is very compact and could be easily fabricated at a low cost. The aperture efficiency of proposed sparse array is significantly better than those of conventional sparse array with identical element and is comparable to that of periodic array.

The scanning performance of proposed array is limited to a relatively small range. This is an extremely unfavorable feature for COTM application. However, the problem is not severe in low latitude regions. Considering the advantage of significant reduction on the active components number which reduces cost and power consumption remarkably, the drawbacks of proposed antenna array are possible to be compensated.

The mutual coupling might bring in unfavorable effects on the performance of sparse array. This is an urgent problem that needs to be studied and tackled on the topic of sparse array researches. 

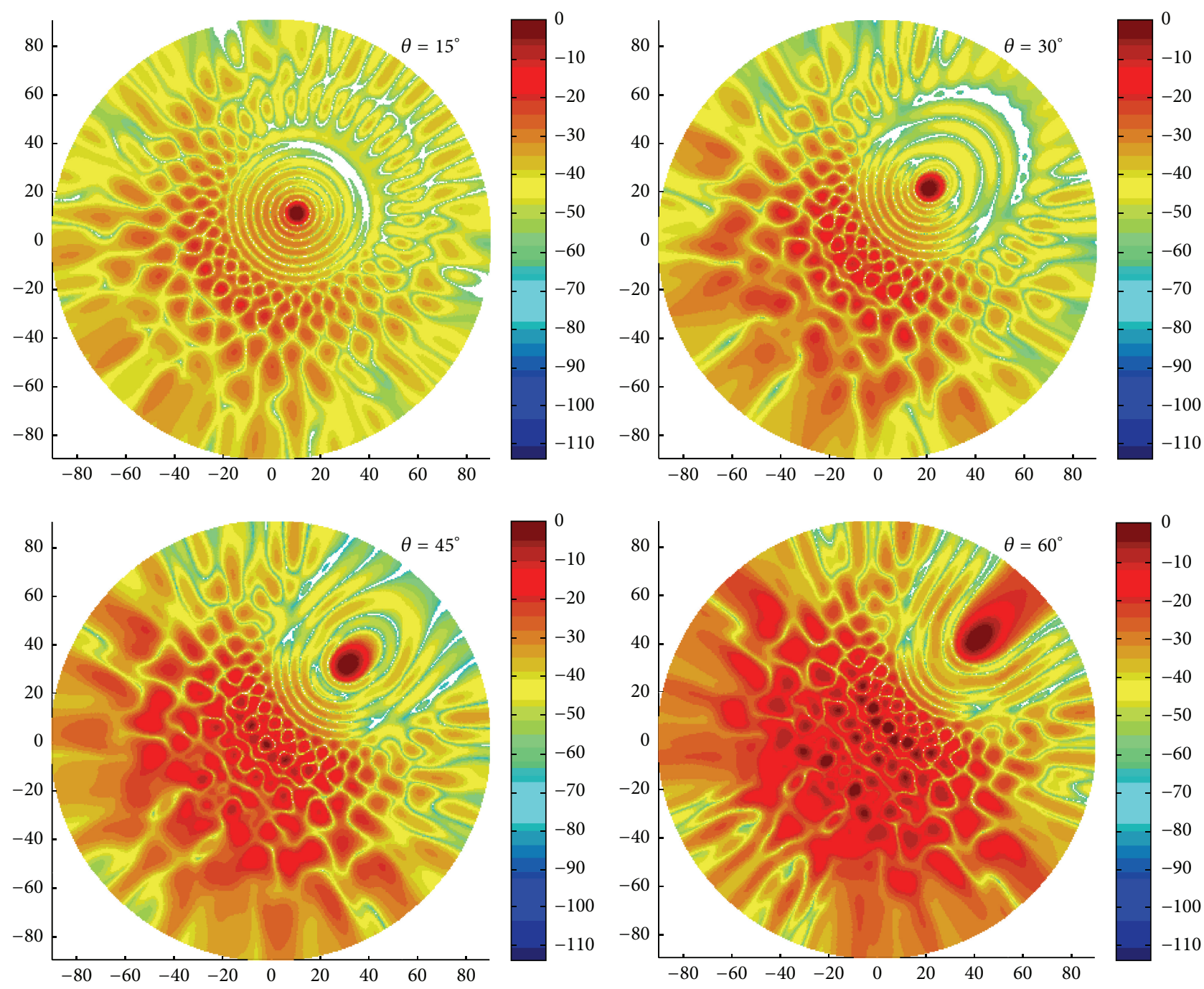

FIGURE 12: Scanning beam pattern of proposed sparse array.

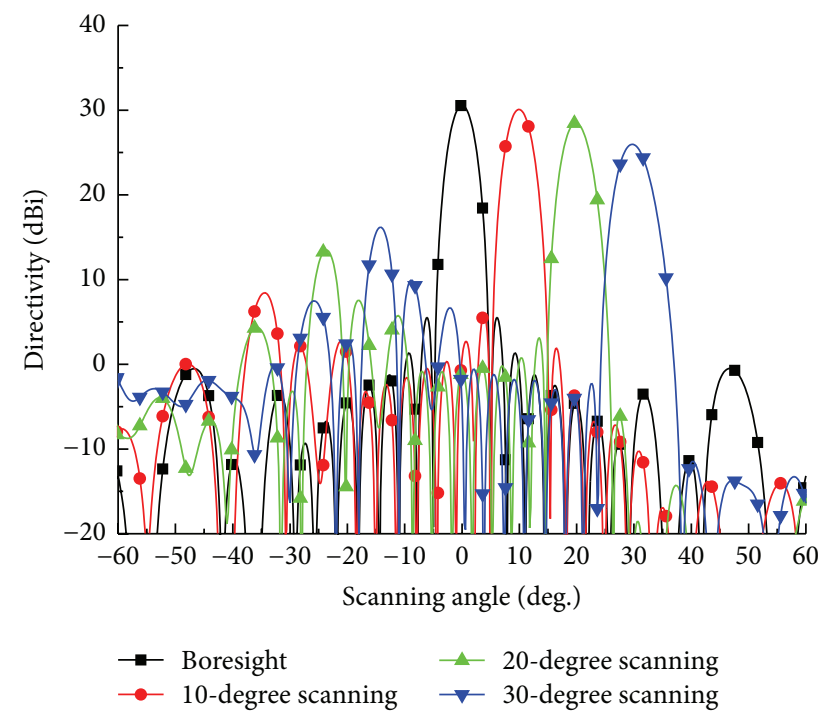

FIGURE 13: Scanning beam of proposed sparse array.

\section{Conflict of Interests}

The authors declare that there is no conflict of interests regarding the publication of this paper.

\section{References}

[1] F. Hodjat and S. A. Hovanessian, "Nonuniformly spaced linear and planar array antennas for sidelobe reduction," IEEE Transactions on Antennas and Propagation, vol. 26, no. 2, pp. 198-204, 1978.

[2] B. P. Kumar and G. R. Branner, "Design of unequally spaced arrays for performance improvement," IEEE Transactions on Antennas and Propagation, vol. 47, no. 3, pp. 511-523, 1999.

[3] R. M. Leahy and B. D. Jeffs, "On the design of maximally sparse beamforming arrays," IEEE Transactions on Antennas and Propagation, vol. 39, no. 8, pp. 1178-1187, 1991.

[4] G. Toso, P. Angeletti, and C. Mangenot, "A comparison of density and amplitude tapering for transmit active arrays," in Proceedings of the 3rd European Conference on Antennas and 
Propagation (EuCAP '09), pp. 840-843, Berlin, Germany, March 2009.

[5] A. Maffett, "Array factors with nonuniform spacing parameter," IRE Transactions on Antennas and Propagation, vol. 10, no. 2, pp. 131-136, 1962.

[6] A. Ishimaru, "Theory of unequally-spaced arrays," IRE Transactions on Antennas and Propagation, vol. 10, no. 6, pp. 691-702, 1962.

[7] L. Zhang, Y.-C. Jiao, B. Chen, and H. Li, "Orthogonal genetic algorithm for planar thinned array designs," International Journal of Antennas and Propagation, vol. 2012, Article ID 319037, 7 pages, 2012.

[8] S. K. Goudos, K. Siakavara, T. Samaras, E. E. Vafiadis, and J. N. Sahalos, "Sparse linear array synthesis with multiple constraints using differential evolution with strategy adaptation," IEEE Antennas and Wireless Propagation Letters, vol. 10, pp. 670-673, 2011.

[9] D. Caratelli and M. C. Viganó, "Analytical synthesis technique for linear uniform-amplitude sparse arrays," Radio Science, vol. 46, no. 4, pp. 1-6, 2011.

[10] M. C. Vigano, G. Toso, and D. Caratelli, "A hybrid deterministic/metaheuristic synthesis technique for non-uniformly spaced linear printed antenna arrays," in Proceedings of the 7th European Conference on Antennas and Propagation, pp. 3650-3653, April 2013.

[11] C. Bencivenni, M. V. Ivashina, R. Maaskant, and J. Wettergren, "Design of maximally sparse antenna arrays in the presence of mutual coupling," IEEE Antennas and Wireless Propagation Letters, vol. 14, pp. 159-162, 2015.

[12] S.-W. Qu, C. H. Chan, M.-Y. Xia, and Z. Nie, "High-efficiency periodic sparse microstrip array based on mutual coupling," IEEE Transactions on Antennas and Propagation, vol. 61, no. 4, pp. 1963-1970, 2013.

[13] M. C. Viganó, G. Toso, G. Caille, C. Mangenot, and I. E. Lager, "Sunflower array antenna with adjustable density taper," International Journal of Antennas and Propagation, vol. 2009, Article ID 624035, 10 pages, 2009.

[14] O. M. Bucci, T. Isernia, A. F. Morabito, S. Perna, and D. Pinchera, "Density and element-size tapering for the design of arrays with a reduced number of control points and high efficiency," in Proceedings of the 4th European Conference on Antennas and Propagation, pp. 1-4, April 2010.

[15] M.-Y. Zheng, K.-S. Chen, H.-G. Wu, and X.-P. Liu, "Sparse planar array synthesis using matrix enhancement and matrix pencil," International Journal of Antennas and Propagation, vol. 2013, Article ID 147097, 7 pages, 2013.

[16] R. Willey, "Space tapaering of linear and planar arrays," IRE Transactions on Antennas and Propagation, vol. 10, no. 4, pp. 369-377, 1962.

[17] Y. Lo and S. Lee, "A study of space-tapered arrays," IEEE Transactions on Antennas and Propagation, vol. 14, no. 1, pp. 2230, 1966.

[18] M. C. Vigano, D. L. del Rio, F. Bongard, and S. Vaccaro, "Sparse array antenna for Ku-band mobile terminals using 1 bit phase controls," IEEE Transactions on Antennas and Propagation, vol. 62, no. 4, pp. 1723-1730, 2014.

[19] P. Angeletti, G. Toso, and G. Ruggerini, "Array antennas with jointly optimized elements positions and dimensions Part II. Planar circular arrays," IEEE Transactions on Antennas and Propagation, vol. 62, no. 4, pp. 1627-1639, 2014.
[20] A. Catalani, L. Russo, O. M. Bucci, T. Isernia, and G. Toso, "Sparse arrays for satellite communications: from optimal design to realization," in Proceedings of the 32nd ESA Antenna Workshop on Antennas for Space Applications, pp. 5-8, Noordwijk, The Netherlands, October 2010.

[21] P. Angeletti and G. Toso, "Array antennas with jointly optimized elements positions and dimensions part I: linear arrays," IEEE Transactions on Antennas and Propagation, vol. 62, no. 4, part 1, pp. 1619-1626, 2014.

[22] L. Baggen, S. Vaccaro, D. Llorens del Rio, J. Padilla, and R. T. Sanchez, "A compact phased array for satcom applications," in Proceedings of the IEEE International Symposium on Phased Array Systems \& Technology, pp. 232-239, Waltham, Mass, USA, October 2013.

[23] G. Ruggerini, "A compact circular horn with high efficiency," in Proceedings of the 4th European Conference on Antennas and Propagation (EuCAP '10), pp. 1-3, IEEE, Barcelona, Spain, April 2010.

[24] J. Granholm and K. Woelders, "Dual polarization stacked microstrip patch antenna array with very low crosspolarization," IEEE Transactions on Antennas and Propagation, vol. 49, no. 10, pp. 1393-1402, 2001. 

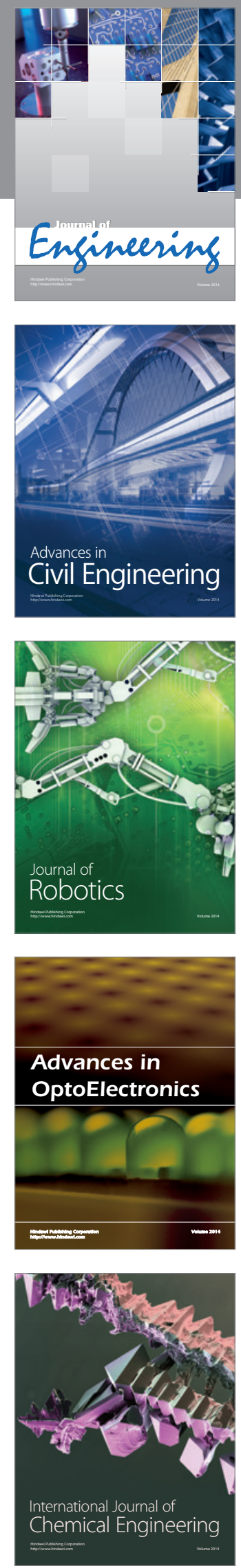

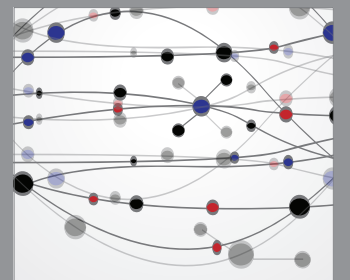

The Scientific World Journal
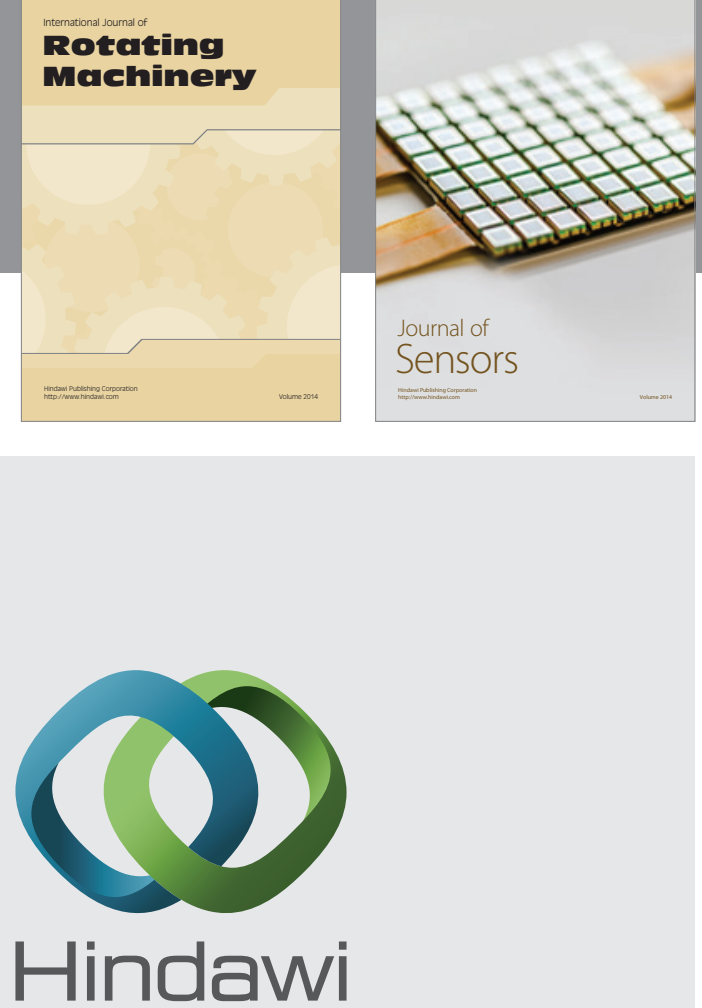

Submit your manuscripts at http://www.hindawi.com
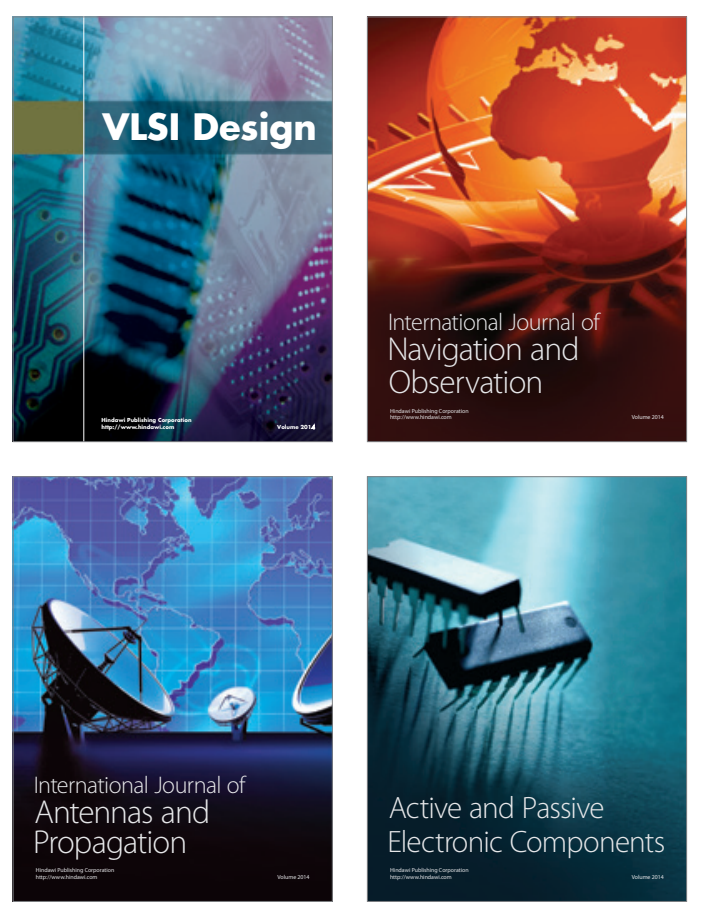
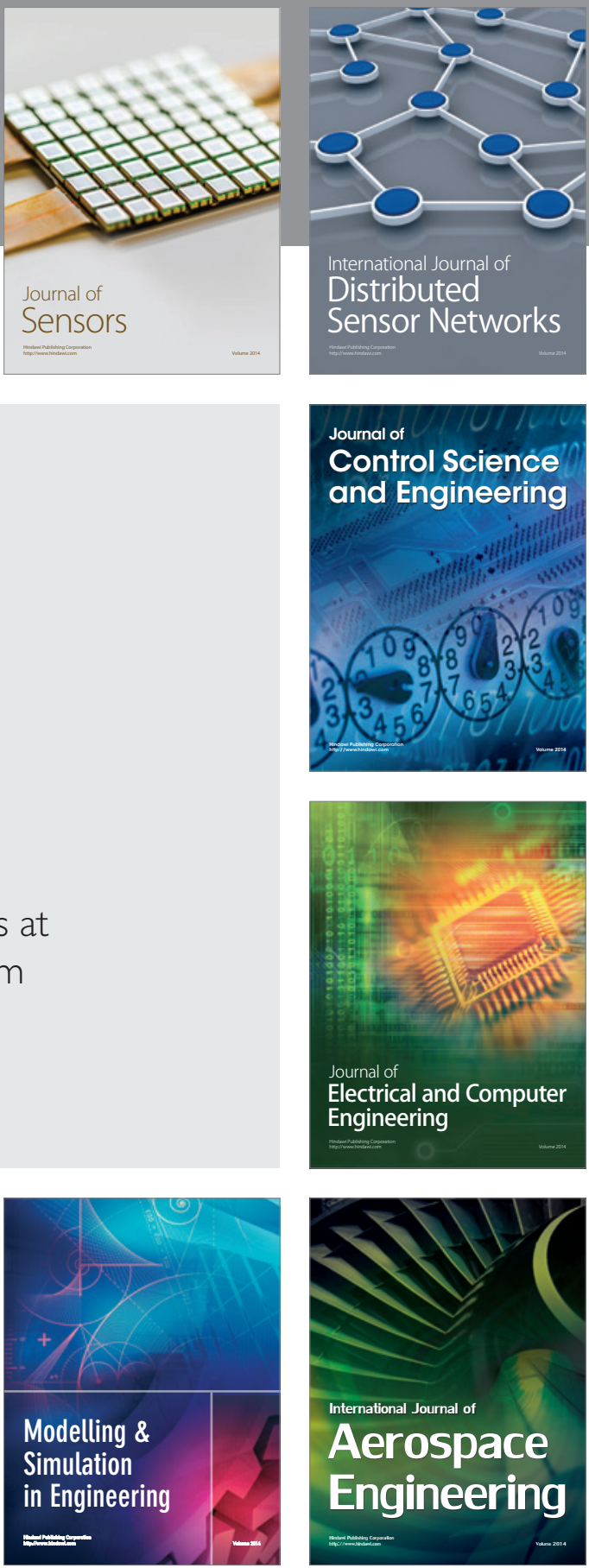

Journal of

Control Science

and Engineering
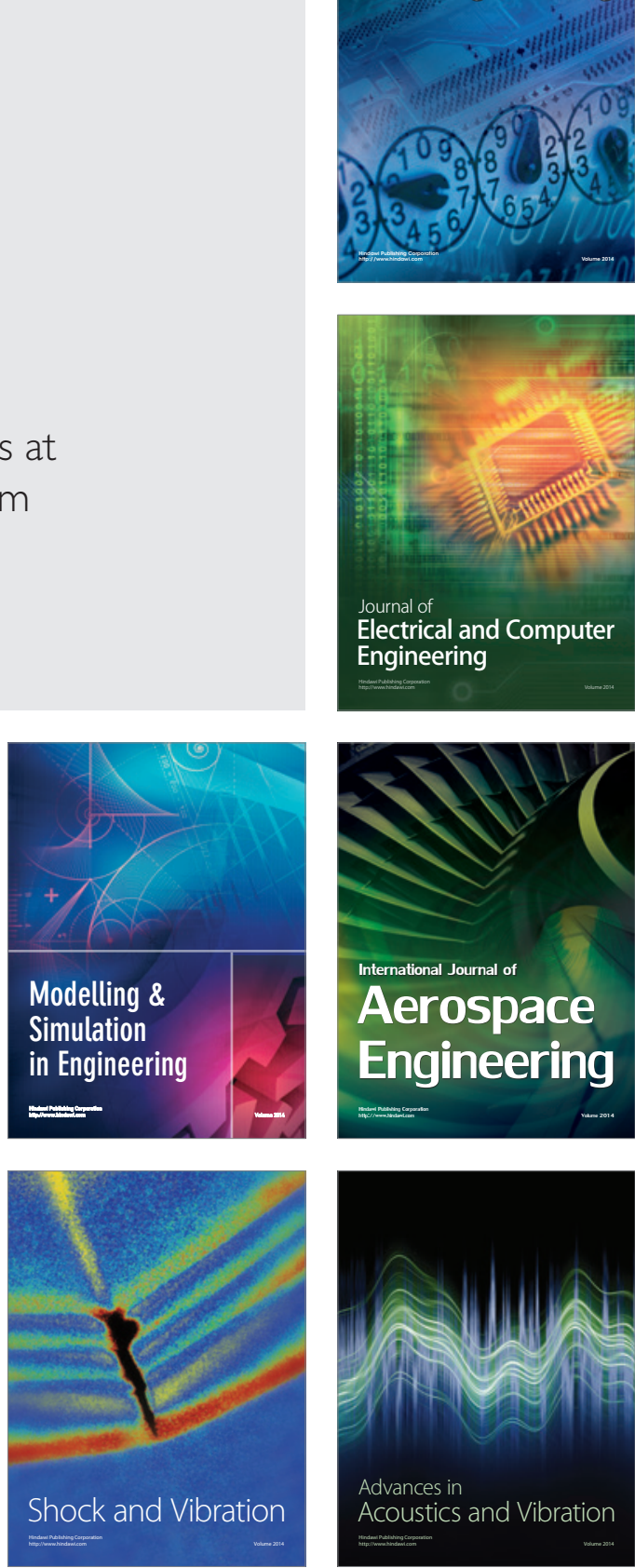